\title{
Brincando na creche: atividades com crianças pequenas ${ }^{1}$
}

\author{
Playing in the creche: activities for young children
}

\author{
Carina de Figueiredo BONOME-PONTOGLIO² \\ Edna Maria MARTURANO
}

\begin{abstract}
Resumo
O objetivo deste estudo é verificar se a exposição da criança a atividades lúdicas mediadas por um adulto na creche favorece sua participação em brincadeiras e interações com outras pessoas. Participaram 12 crianças de creche, de ambos os sexos, com idade entre 22 e 26 meses. Foram realizadas 20 sessões coletivas de atividades lúdicas, três vezes por semana. As crianças foram observadas antes e depois da intervenção. Os registros de observação foram analisados quanto à participação em brincadeira e quanto ao comportamento orientado para o ambiente. As medidas pré e pós-intervenção foram comparadas por meio do teste de Wilcoxon. Nessas comparações, houve diminuição da brincadeira solitária, aumento da brincadeira paralela e tendência ao aumento da brincadeira associativa. A interação positiva com criança aumentou e a interação negativa com adulto tendeu para uma redução. A pesquisa traz contribuição para práticas de promoção do desenvolvimento da criança pequena em contexto de creche.
\end{abstract}

Unitermos: Creche. Desenvolvimento infantil. Recreação.

\begin{abstract}
This aim of this study was to ascertain ifexposure to adult-mediated play activities in a crèche would improve children's participation in play and in social interaction. Twelve children of both sexes, aged from 22 to 26 months, participated in 20 collective play activity sessions, three times a week. We observed the children before and after the intervention and analyzed the observation records in terms of children's participation in play and environment-oriented behavior. By means of comparisons between pre-and post-intervention measures using the Wilcoxon Test, we detected a reduction in solitary play and an increase in associative play. Positive interaction with peers increased, while negative interaction with adults tended to be reduced. The research contributes to practices that promote the development of young children in crèches.
\end{abstract}

Uniterms: Child day care. Childhood development. Recreation.

Avanços recentes no campo das neurociências contribuíram para a compreensão do desenvolvimento da criança pequena em suas interações com o ambiente.
A evidência acumulada reitera a notável plasticidade do cérebro humano nos primeiros anos de vida (Shore, 2000). Com isso, impõe-se a noção de que os cuidados

$\operatorname{coth}$

1 Artigo elaborado a partir da dissertação de C.F. BONOME-PONTOGLIO, intitulada "Brincando na creche: atividades com crianças pequenas". Universidade de São Paulo, 2009.

2 Educadora musical. Ribeirão Preto, SP, Brasil.

3 Universidade de São Paulo, Faculdade de Medicina de Ribeirão Preto, Departamento de Neurociências e Ciências do Comportamento. R. Tenente Catão Roxo, 2650, Bloco Saúde Mental,14051-140, Ribeirão Preto, SP, Brasil. Correspondência para/Correspondence to: E.M. MARTURANO. E-mail: <emmartur@fmrp.usp.br>. 
precoces têm um impacto decisivo e duradouro no desenvolvimento (Shore, 2000). A creche como contexto de desenvolvimento ganha destaque, visto que, com a participação crescente das mulheres no mercado de trabalho, cresce também a demanda por cuidados alternativos para bebês e crianças pequenas.

No Brasil, o atendimento em creches passa por uma fase de transição. Foram estabelecidas as balizas oficiais para uma adequação do sistema à tarefa educativa (Brasil, 1996; 1998). No entanto, na prática, a efetivação dessa meta esbarra em obstáculos relacionados a uma cultura dominante de "cuidados" (Lordelo, 1998; Vitta \& Emmel, 2004) e a deficiências na formação do educador para o trabalho com crianças pequenas (Campos, Fullgraf \& Wiggers, 2006; Carvalho et al., 2006). Esse é um panorama preocupante, pois a creche tem impacto no desenvolvimento da criança e a qualidade do atendimento é um diferencial importante na direção dessa influência.

A avaliação do impacto da creche no desenvolvimento apresenta resultados mistos. Há evidência de que programas de qualidade influenciem positivamente as habilidades cognitivas globais, a linguagem e o desempenho escolar da criança, com efeito maior em crianças de famílias pobres (Bee, 2003; Penn et al., 2006). Crianças de creche tendem a ser mais sociáveis e mais populares com os companheiros em relação àquelas criadas em casa; elas mostram habilidades sociais mais desenvolvidas, brincadeiras com pares mais avançadas e maior conhecimento das regras sociais. Mas também foi encontrado que crianças com experiência de creche se tornaram posteriormente mais agressivas com os pares e mais desobedientes em relação aos adultos (Bee, 2003; NICHD, 2004).

Pesquisas brasileiras também apresentam resultados mistos. Estudos longitudinais com crianças de até três anos não detectaram associação entre permanência na creche e desenvolvimento cognitivo (Lordelo, Chalhub, Guirra \& Carvalho, 2007) ou da linguagem (Rezende, Lima, Beteli \& Santos, 2003). Encontrou-se, porém, um percentual de crianças menor que o esperado para os 9-12 meses com comportamentos de linguagem típicos da fase, especificamente ter falado a primeira palavra, iniciar jogos gestuais e seguir ordem com gesto (Lima, Barbarini, Gagliardo, Arnais \& Gonçalves, 2004). Crianças de creches públicas, comparadas a crianças de creches particulares, apresentaram atraso na linguagem, atribuído à menor escolaridade dos educadores e das mães (Ramos, Pedromônico, Shinzato \& Lucas, 2002).

Na tentativa de integrar resultados divergentes, sugere-se que o mais importante não é o fato de a criança frequentar creche ou não, e sim a qualidade do ambiente. O que deve ser foco da análise são as experiências vividas pela criança na creche (Hynes \& Tara Habasevich-Brooks, 2008; Souza \& Campos-de-Carvalho, 2005). De todo modo, a permanência no ambiente da creche tem consequências que podem ser positivas ou negativas para o desenvolvimento da criança e que afetam mais amplamente o domínio da sociabilidade (Lordelo, 2002).

Tendo em vista as oportunidades de desenvolvimento da sociabilidade que o ambiente da creche proporciona (Vasconcelos \& Ferreira, 2002), avalia-se neste artigo uma intervenção para amplificar tais oportunidades por meio de atividades lúdicas. Foi escolhida como alvo a idade de 24 meses, em torno da qual há uma expansão da capacidade adaptativa, do potencial de aprendizagem e das habilidades interpessoais da criança (Bee, 2003; M. Cole \& S. R. Cole, 2004).

Uma importante conquista cognitiva nessa fase é a capacidade de basear as ações em símbolos mentais: representações de experiências anteriores (M. Cole \& S. R. Cole, 2004). No domínio da linguagem, aparecem as primeiras frases de duas palavras; ao redor dos 21 meses de idade, as crianças já são capazes de seguir instruções verbais relativamente complexas (M. Cole \& S. R. Cole, 2004).

A convergência de aquisições cognitivas e de linguagem está associada a avanços nas habilidades interpessoais e maior complexidade nas interações (Brownell, 1986; Eckerman \& Didow, 1996). Observações em contexto de creche, com crianças de 11 e 24 meses, sugerem que o maior tempo ocupado pelas crianças mais velhas em interações com os pares se deve ao maior uso da linguagem oral pelas crianças de dois anos (Finkelstein, Dent, Gallacher \& Ramey, 1978). A sociabilidade é também influenciada pela experiência. Bebês de nove meses já revelam atenção compartilhada, o que reflete sua tendência para se orientarem socialmente enquanto observam um objeto ou um evento (Lampreia, 2007); nessa idade, há indícios de que a mera 
exposição à presença de outros bebês aumente a frequência do comportamento orientado para os pares (Becker, 1977). Na mesma direção, foi observado que bebês que se conhecem se tornam mais sociáveis à medida que participam de interações uns com os outros (Mueller \& Brenner, 1977).

Nas brincadeiras emerge o faz-de-conta, quando um objeto representa outro (M. Cole \& S. R.Cole, 2004). Também o compartilhamento das brincadeiras aumenta e se torna mais complexo. Em um estudo clássico, Parten (1932) definiu categorias de participação social com base na observação de brincadeiras espontâneas de crianças, definindo níveis de participação associados com o desenvolvimento. A categoria de participação mais frequentemente observada foi a brincadeira paralela, em que a criança brinca perto de outras, com brinquedos semelhantes, mas não tenta influenciar ou modificar a atividade das demais. Dos dois aos quatro anos, a brincadeira solitária e a brincadeira paralela diminuem. Ao mesmo tempo, aumentam formas mais avançadas de brincadeira em grupo: a brincadeira associativa e a cooperativa:

Observações recentes no contexto da creche confirmam a tendência etária de diminuição da brincadeira individual e aumento da brincadeira em grupo (Lordelo \& Carvalho, 2006). A brincadeira paralela, por sua vez, tem sido apontada como um contexto facilitador de relações entre as crianças (Mueller \& Brenner, 1977). Um componente importante da brincadeira nessa fase é a imitação.

Por volta dos 14 meses, os bebês conseguem aprender uns com os outros por meio da imitação adiada de ações simples (Hanna \& Meltzoff, 1993). Entre 15 e 19 meses, desenvolvem a capacidade de imitar, de memória, sequências de duas ações (Fenson \& Ramsay, 1981). A capacidade de reproduzir comportamentos cada vez mais complexos, aliada ao grande interesse das crianças em observar e imitar outras pessoas, faz da imitação um importante recurso de expansão das habilidades (Eckerman \& Didow, 1996).

As mudanças típicas do final do segundo ano de vida refletem a emergência de várias capacidades novas. O pressuposto principal da intervenção avaliada neste artigo é de que o desenvolvimento de capacidades emergentes pode ser favorecido com uma intervenção apropriada na zona de desenvolvimento proximal, definida por Vygotsky (2000, p.112) como "a distância entre o nível de desenvolvimento real, que se costuma determinar através da solução independente de problemas, e o nível de desenvolvimento potencial, determinado por meio da solução de problemas sob a orientação de um adulto ou em colaboração com companheiros mais capazes". Com base nas proposições de Vygotsky (2000), supõe-se que as crianças aprendam e se desenvolvam nas interações sociais, e que a imitação e a brincadeira são instâncias em que elas funcionam na zona de desenvolvimento proximal (Palangana, 2001; Queiroz, Maciel \& Branco, 2006).

O estudo tem como objetivo verificar se a exposição a atividades regulares de brincadeira em conjunto, mediadas por um adulto, favorece o desenvolvimento da criança pequena no contexto de creche em termos de aumento na participação em brincadeiras, aumento das interações positivas com outras pessoas, crianças ou adultos, e diminuição das interações negativas com outras pessoas.

\section{Método}

\section{Participantes}

A pesquisa foi conduzida em uma creche filantrópica conveniada com a Prefeitura Municipal de Ribeirão Preto (SP), tendo dela participado uma turma de 17 crianças, monitorada por dois educadores. Cinco crianças saíram da creche depois de iniciado o estudo, que foi concluído, portanto, com 12 crianças (3 meninas e 9 meninos) com idade entre 22 e 26 meses (média 24 meses) no início do estudo.

\section{Procedimentos}

O projeto foi aprovado pelo Comitê de Ética em Pesquisa da Faculdade de Filosofia, Ciências e Letras de Ribeirão Preto da Universidade de São Paulo, protocolo n²72/2006, em 23/11/2006, e os responsáveis por todos os participantes assinaram um Termo de Consentimento Livre e Esclarecido antes de sua inclusão na amostra. Uma vez aprovado, o projeto foi apresentado aos pais em reunião agendada pela direção da creche. Todos os pais concordaram com a participação de seus filhos. 
Antes de iniciar a coleta de dados, foi realizado um estudo-piloto com outra turma, tendo por finalidade verificar a exequibilidade da intervenção no contexto da creche, definir o procedimento de observação, obter registros de comportamentos que serviriam como base para a construção das categorias de brincadeira e de comportamento a serem empregadas na análise dos dados e treinar uma auxiliar de pesquisa.

Antes da intervenção, as crianças foram observadas, em média, oito vezes durante os meses de março e abril de 2007. Foi adotado o registro cursivo em intervalos de tempo: de acordo com uma ordem aleatória previamente estabelecida, cada criança era observada durante cinco minutos. Era feito um rodízio completo de todos os participantes em cada sessão, com duração aproximada de 60 minutos. As observações foram realizadas pela auxiliar de pesquisa.

O programa de atividades lúdicas foi conduzido pela primeira autora, em 20 sessões temáticas, com duração de 15 a 30 minutos, ao longo de dois meses. Foram trabalhados temas relacionados a animais, uso de objetos, tradições culturais, conhecimento do próprio corpo (Brasil, 1998). O tema da sessão era introduzido com uma cantiga que as crianças cantavam, ora imitando a mímica da educadora, ora realizando ações de acordo com a letra da cantiga. Na sequencia, participavam de brincadeira ou dramatização. Tanto na cantiga como na brincadeira, era incentivado o contato entre as crianças por meio de ações simples, como dar e receber, abraçar, alternar sua vez. As atividades tinham apoio em objetos confeccionados com material de baixo custo (máscaras, instrumentos musicais, bichinhos de origami, etc.).

As sessões eram realizadas com todas as crianças presentes (entre 13 e 17), no ambiente onde a turma estava instalada. Os educadores responsáveis pela turma permaneciam na sala; eram instruídos para apenas participar junto com as crianças, seguindo as mesmas instruções dadas a elas, somente participando de outra forma quando solicitados. Das 12 crianças participantes do estudo, três estiveram presentes em todas as sessões de atividades lúdicas, seis participaram de 19 sessões, duas participaram de 17 e apenas uma criança participou de 15 sessões.

Na semana seguinte à última sessão de intervenção, foram iniciadas as sessões de observação pós-intervenção. As observações foram realizadas duas a três vezes por semana. Para cada criança, foi completado o mesmo número de observações feitas na fase pré-intervenção.

Para a análise dos dados, foram feitas duas leituras sobre os registros de observação. Uma delas tinha como foco a participação da criança em brincadeiras, e a outra tinha a finalidade de categorizar o comportamento da criança em relação às pessoas e ao ambiente físico.

Categorias de brincadeiras: foram utilizadas as seguintes categorias de brincadeiras derivadas do sistema proposto por Parten (1932):

- Ausência de brincadeira: quando a criança não parece estar brincando; essa categoria inclui as categorias desocupado e observador de Parten.

- Brincadeira solitária: quando a criança brinca sozinha, seja com algum objeto, seja com seu próprio corpo, e não estabelece nenhuma interação social relacionada ao conteúdo e propósito da brincadeira, nem faz qualquer esforço para se aproximar de outras crianças.

- Brincadeira paralela: quando a criança brinca próxima a um ou mais companheiros, com brinquedos semelhantes ou sobre um mesmo tema, porém de maneira independente e não necessariamente igual, sem interagir quanto à brincadeira e sem tentar influenciar o brincar do outro.

- Brincadeira associativa: quando duas ou mais crianças brincam juntas, de modo semelhante, sobre o mesmo tema; elas interagem, seguem umas às outras, emprestam e tomam emprestados brinquedos, porém não há divisão de tarefas e nenhuma organização.

- Brincadeira cooperativa: quando há, na brincadeira de duas ou mais crianças sobre um mesmo tema, cooperação ou competição, divisão de tarefas e de papéis.

Categorias de comportamento - Com base nos trabalhos de Bussab e Maluf (1998) e Meneghini e Campos de Carvalho (2003), foram construídas onze categorias comportamentais, compreendendo 23 subcategorias. As categorias maiores e suas respectivas definições são apresentadas resumidamente:

- Atividade individual: a criança se entretém sozinha, em contato ativo com alguma parte de seu próprio corpo ou com um objeto. 
- Choro: a criança começa a chorar sem causa identificada ou por um motivo que não seja uma agressão, como, por exemplo, a saída do educador da sala.

- Comportamento socialmente dirigido: a criança olha para outra pessoa, adulto ou criança, e concomitante ou imediatamente após emite um comportamento (verbaliza, toca, sorri), sem ocorrência de resposta do outro.

- Defender-se sem agredir o outro: a criança se defende de outra criança que a agride ou disputa com ela, mas sem agredir a outra, e, por exemplo, chamando a educadora.

- Ignorar contato de adulto: a criança não responde a uma tentativa de interação realizada por um adulto.

- Ignorar contato de criança: a criança não responde a uma tentativa de interação realizada por outra criança; por exemplo, não responde ao toque ou ao chamado.

- Interação negativa com adulto: a criança exibe contato não amistoso com adulto: briga, agressão, disputa, birra ou desobedece a uma ordem ou instrução.

- Interação negativa com criança: inclui comportamentos de agressão dirigidos a outra criança, como falar brava, empurrar, bater, tentar morder; disputar um objeto ou a atenção do adulto; chorar ou revidar em resposta a uma agressão.

- Interação positiva com adulto: a criança participa de algum tipo de troca com um adulto, como brincadeira, conversa, olhar recíproco; atende a uma ordem ou instrução dada pelo educador; coopera, pegando, por exemplo, espontaneamente um objeto que caiu no chão e entregando-o para a pessoa.

- Interação positiva com criança: a criança participa de algum tipo de troca com outra criança: brincadeira, conversa, olhar recíproco.

Observação: registrada quando a criança não está engajada em atividade e olha com atenção para um foco identificável: adulto, criança, grupo de crianças ou um local definido no ambiente.

A análise dos protocolos para caracterização da brincadeira foi feita pela primeira autora e a auxiliar de pesquisa. Os registros de observação de todas as crianças foram analisados pelas duas avaliadoras. Para cada criança foi calculado um índice de acordo com base na fórmula [(acordos - desacordos)/total de brincadeiras categorizadas no protocolo $\times 100]$. A referência para o denominador na fórmula era a categorização feita pela pesquisadora. Os índices obtidos variaram entre $85 \%$ e 95\%. Os desacordos foram discutidos e resolvidos por consenso.

A auxiliar de pesquisa aplicou as categorias comportamentais aos registros de observação, após a verificação do acordo, feita sobre os registros de duas sessões. Os índices de acordo para o conjunto de onze categorias foram $77 \%$ e $81 \%$.

A frequência de cada categoria foi computada, por período de observação, para cada criança. Os dados de frequência do período pré-intervenção foram comparados com os do período pós-intervenção por meio do Teste de Wilcoxon para amostras dependentes. Foram considerados significativos os resultados com $p \leq 0,05$.

\section{Resultados}

No que concerne às categorias de brincadeira, todas as crianças já apresentam Brincadeira Solitária nas observações iniciais: um resultado que se mantém depois da intervenção. Ausência de Brincadeira também aparece na observação de todas as crianças, em ambos os períodos. Cinco crianças participam de Brincadeira Paralela antes da intervenção; esse número sobe para oito depois da intervenção. A Brincadeira Associativa, observada na atividade de oito crianças antes da intervenção, passa a ser exibida por onze no período pós-intervenção. Apenas uma criança foi observada em Brincadeira Cooperativa em ambos os períodos de observação; uma criança passa a apresentar essa categoria de brincadeira depois da intervenção.

A (Tabela 1) apresenta os resultados de frequência referentes às categorias de brincadeira, antes e depois da intervenção. Na tabela também se encontra o número de crianças que aumentaram ou diminuíram sua participação em cada tipo de brincadeira, entre as avaliações pré e pós-intervenção. Observa-se que Ausência de Brincadeira e Brincadeira Solitária são as categorias com maior frequência média nos dois períodos de observação.

A comparação entre os dados de frequência dos períodos pré e pós-intervenção, por meio do Teste de Wilcoxon, detectou duas variações significativas: onze 
crianças passam a exibir com menor frequência a Brincadeira Solitária, ao passo que oito aumentam a frequência de Brincadeira Paralela. Também a Brincadeira Associativa tende a aumentar $(p<0,10)$ depois da intervenção. Desse modo, a brincadeira predominante na amostra é a solitária, porém com tendência ao decréscimo. Por outro lado, formas mais avançadas de brincadeira, como a paralela e a associativa, aparecem com menor frequência, mas com clara tendência ascendente tanto em termos de frequência média como em termos do número de crianças que as exibem.

Observa-se (Tabela 2) que cinco dentre as onze categorias comportamentais são bastante frequentes no período pré-intervenção: Atividade Individual, Observação, Interação Positiva com Adulto, Comportamento Socialmente Dirigido, Interação Positiva com Criança. Dados não apresentados na tabela indicam que essas categorias são exibidas, desde o início, por pelo menos dez crianças. Depois da intervenção, esses comportamentos continuam sendo os mais frequentes.
A comparação entre os dados de frequência dos períodos pré e pós-intervenção, por meio do Teste de Wilcoxon, detectou duas variações significativas $(p<0,05)$ : depois da intervenção, aumentou a frequência de Interação Positiva com Criança, ao passo que Atividade Individual diminuiu. As categorias Interação Negativa com Adulto e Ignorar Contato de Adulto mostram tendência à diminuição $(p<0,10)$. Em termos de variação nominal (significativa ou não), observa-se maior número de crianças diminuindo, por um lado, a Atividade Individual, o Comportamento Socialmente Dirigido, o Ignorar Contato de Adulto e a Interação Negativa com Adulto, e aumentando, por outro, a Interação Positiva com Adulto e a Interação Positiva com Criança.

\section{Discussão}

Este estudo teve como objetivo verificar se crianças pequenas, quando expostas a brincadeiras

Tabela 1.Categorias de brincadeira antes e depois da intervenção: médias e número de crianças que aumentaram ou diminuíram a frequência da categoria.

\begin{tabular}{|c|c|c|c|c|c|c|}
\hline \multirow{2}{*}{ Categorias de brincadeira } & \multicolumn{2}{|c|}{ Médias } & \multicolumn{2}{|c|}{ Variação pré-pós } & \multirow{2}{*}{$Z$} & \multirow{2}{*}{$p$} \\
\hline & Pré & Pós & Aumento & Redução & & \\
\hline Ausência de brincadeira & 14,67 & 13,17 & 4 & 5 & 0,712 & 0,476 \\
\hline Brincadeira solitária & 24,00 & 14,17 & 1 & 11 & 2,867 & 0,004 \\
\hline Brincadeira paralela & 0,42 & 1,83 & 8 & 2 & 2,140 & 0,032 \\
\hline Brincadeira associativa & 2,58 & 4,25 & 8 & 2 & 1,740 & 0,082 \\
\hline Brincadeira cooperativa & 0,17 & 0,33 & 2 & 0 & 1,414 & 0,157 \\
\hline
\end{tabular}

Nota: Teste de Wilcoxon. Pré: antes da intervenção. Pós: depois da intervenção.

Tabela 2. Categorias comportamentais antes e depois da intervenção: médias e número de crianças que aumentaram ou diminuíram a frequência da categoria.

\begin{tabular}{|c|c|c|c|c|c|c|}
\hline \multirow{2}{*}{ Categorias comportamentais } & \multicolumn{2}{|c|}{ Médias } & \multicolumn{2}{|c|}{ Variação pré-pós } & \multirow{2}{*}{ Z } & \multirow{2}{*}{$p$} \\
\hline & Pré & Pós & Aumento & Redução & & \\
\hline Atividade individual & 23,83 & 16,50 & 0 & 12 & 3,066 & 0,002 \\
\hline Choro & 0,33 & 0,42 & 3 & 2 & 0,447 & 0,655 \\
\hline Comportamento socialmente dirigido & 4,83 & 3,50 & 3 & 7 & 1,283 & 0,199 \\
\hline Defender-se sem agredir o outro & 0,25 & 0,42 & 3 & 1 & 0,557 & 0,577 \\
\hline Ignorar contato de adulto & 0,67 & 0,08 & 1 & 6 & 1,897 & 0,058 \\
\hline Ignorar contato de criança & 0,17 & 0,25 & 2 & 1 & 0,577 & 0,564 \\
\hline Interação negativa com adulto & 2,00 & 1,08 & 3 & 8 & 1,724 & 0,085 \\
\hline Interação negativa com criança & 1,92 & 2,00 & 4 & 4 & 0,071 & 0,944 \\
\hline Interação positiva com adulto & 11,67 & 13,25 & 8 & 2 & 1,382 & 0,167 \\
\hline Interação positiva com criança & 4,25 & 8,67 & 10 & 1 & 2,671 & 0,008 \\
\hline Observação & 14,58 & 14,92 & 7 & 5 & 0,197 & 0,844 \\
\hline
\end{tabular}


mediadas pelo adulto no contexto da creche, são favorecidas em seu desenvolvimento em relação à brincadeira e às interações com crianças e adultos. Esperava-se que a participação em um programa de atividades lúdicas propiciasse aumento do envolvimento em brincadeiras com outras crianças. Também se esperava aumento das interações positivas e diminuição das interações negativas. Por meio de comparações entre comportamentos observados antes e depois da intervenção, alguns dos resultados esperados foram efetivamente obtidos. Houve diminuição da brincadeira solitária, aumento da brincadeira paralela e tendência ao aumento da brincadeira associativa. A interação positiva com criança aumentou e a interação negativa com adulto tendeu para uma redução.

O padrão de mudanças corresponde a uma tendência de desenvolvimento (Finkelstein et al., 1978; Lordelo \& Carvalho, 2006; Parten, 1932). Apesar de predominar ainda a brincadeira solitária, a intervenção parece ter favorecido o desenvolvimento de um brincar mais social. Esses resultados podem ser interpretados à luz das proposições de Vygotsky (2000): o adulto, agindo na zona do desenvolvimento proximal, propiciou experiências guiadas, por meio das quais um desenvolvimento potencial se atualizou em desenvolvimento real, ampliando o repertório das crianças no que diz respeito às interações e à brincadeira social. Não temos elementos para identificar os ingredientes da intervenção que contribuíram para esses resultados; no entanto, pode-se especular a esse respeito.

Uma característica relevante da intervenção é o apoio na imitação; o tempo todo, o adulto ofereceu modelos para as crianças imitarem. Além disso, tratava-se de atividades coletivas, em que a criança tinha a oportunidade de observar companheiros mais proficientes. Há evidência de que no segundo ano de vida as crianças exibem brincadeira mais avançada depois da observação de modelos (Fenson \& Ramsay, 1981), e que a atividade imitativa contribui para o uso de estratégias verbais de coordenação de ações na brincadeira (Eckerman \& Didow, 1996).

Outra característica da intervenção que pode ter contribuído decisivamente para os resultados é o estímulo à interação. A experiência de interagir cotidianamente com os pares favorece o desenvolvimento de habilidades sociais da criança pequena (Mueller \&
Brenner, 1977). O estímulo à interação, em ciclos de brincadeira com alternância de ações coordenadas entre as crianças, configura um trabalho na zona de desenvolvimento proximal entre a capacidade de realizar ações orientadas para os pares, já estabelecida (Becker, 1977), e a capacidade de sustentar interações envolvendo reciprocidade, em vias de desenvolvimento (Finkelstein et al., 1978).

$\mathrm{Na}$ amostra do presente estudo, tanto os comportamentos socialmente dirigidos como as interações já estavam presentes no repertório das crianças antes da intervenção. No entanto, depois da intervenção, as ações socialmente dirigidas tendem a diminuir, ao passo que as interações envolvendo trocas aumentam significativamente.

O incentivo à interação, da forma como foi efetivado nas sessões de intervenção, pode ter favorecido o uso da linguagem, uma área vulnerável a atraso no desenvolvimento de crianças que frequentam creche (Lima et al., 2004; Ramos et al., 2002). Se a intervenção favoreceu o uso da linguagem oral, isso também pode ter contribuído para o aumento nas interações positivas entre as crianças, pois o uso da linguagem oral é um fator influente nesse aumento (Finkelstein et al., 1978).

O estudo tem limitações metodológicas que devem ser levadas em conta na apreciação dos seus resultados. O procedimento de observação, sem apoio em recursos de gravação de imagem, é talvez a limitação mais importante por afetar diretamente a qualidade dos dados. O procedimento de registro manual pode ter contribuído para uma subestimação das mudanças comportamentais verificadas entre a avaliação pré-intervenção e a avaliação pós-intervenção, já que a observação"a olho nu"está sujeita a perdas, o que reduz a sensibilidade do procedimento em relação à gravação de imagem para posterior análise.

Além disso, o desenho do estudo não permite concluir que as mudanças observadas se devam à intervenção, já que estavam operando simultaneamente os processos regulares de desenvolvimento e as influências presentes no contexto da creche. Nesse sentido, Parten (1932) já havia observado diminuição de atividade solitária e aumento de brincadeira associativa e cooperativa na creche, sem intervenção, ao longo de sete meses - um período maior que os dois meses que distaram entre as avaliações pré- e pós-intervenção no presente estudo. 
Não obstante as limitações assinaladas, os resultados apontaram na direção esperada e têm implicações para práticas de promoção do desenvolvimento. A intervenção teve resultados positivos, nas condições reais de trabalho proporcionadas pela creche e, por utilizar apenas material de baixo custo e não necessitar de um ambiente especial, pode ser facilmente implementada. Da forma como foi concebida, pode ser vista como um conjunto de atividades educativas, coerente com o Referencial Curricular Nacional para a Educação Infantil (Brasil, 1998), que trabalha com temas relativos à formação pessoal e social e ao conhecimento de mundo.

A proposta de intervenção deve ser entendida como um conjunto de atividades pedagógicas de natureza lúdica e não como promoção da brincadeira em si (Queiroz et al., 2006). Essa distinção é importante uma vez que não se está oportunizando a brincadeira livre, que também é um recurso relevante para o desenvolvimento de novas habilidades, mas sim capitalizando o potencial motivador e enriquecedor da brincadeira, de modo a promover atividades que ao mesmo tempo envolvam as crianças e impulsionem seu desenvolvimento.

A implementação de um programa como o apresentado neste estudo poderia ser dificultada por algumas das condições que caracterizam a realidade das creches. Como visto na introdução, a rede de creches no Brasil passa por uma fase de transição, em que as práticas ainda não foram adequadas à legislação que atribuiu à creche o status de instituição educativa (Brasil, 1996; 1998; (ampos et al., 2006).

Para a adoção de atividades educativas planejadas, a cultura de "cuidados" precisa ser superada, embora se reconheça que as situações de cuidados propiciem ricas e variadas oportunidades de interação promotora do desenvolvimento (Brasil, 1998; Seabra \& Seidl de Moura, 2005). Profissionais adaptados a essa cultura talvez mostrem resistência a mudanças, demandando um trabalho prévio de sensibilização.

Outra condição que pode obstruir a implementação de uma intervenção como a proposta neste estudo é a da administração do tempo do educador. Como apontado por Lordelo (1998), a maior parte do tempo dos profissionais de creche é investida em atividades de cuidados físicos, controle e tarefas que não estão diretamente relacionadas ao trabalho com a criança. Para promover atividades sistemáticas de mediação, o educador precisa do apoio da administração da creche para dedicar mais tempo ao contato direto com as crianças, além dos momentos dedicados ao cuidado.

Os resultados do presente estudo sugerem que, com crianças de dois anos, trinta minutos diários de atividades educativas lúdicas com a mediação do adulto podem ser suficientes para promover o desenvolvimento de habilidades; no restante do período reservado à recreação, as crianças têm a oportunidade de exercitar os comportamentos recém-aprendidos e construir sobre eles novas capacidades por meio da brincadeira espontânea não regulada pelo adulto (Queiroz et al., 2006). Nesses momentos de brincadeira livre, é importante que as crianças possam interagir com colegas mais velhos e mais jovens (Brasil, 1998), já que a convivência entre crianças de diferentes faixas etárias propicia experiências de aprendizagem.

\section{Referências}

Becker, J.M.T. (1977). A Learning analysis of the development of peer-oriented behavior in nine-month-old infants. Developmental Psychogoly, 13 (5), 481-491.

Bee, H. (2003). A criança em desenvolvimento (9a ed). Porto Alegre: Artmed.

Brasil. (1996). Lei Federal no 9.394, de 26 de dezembro de 1996. (1996, 23 de dezembro) Lei de Diretrizes e Bases da Educação Nacional. Diário Oficial da União.

Brasil, Ministério da Educação, Coordenação de Educação Infantil. (1998). Referencial curricularnacional para a educação Infantil (Vol. 3). Brasília: MEC.

Brownell, C. A. (1986). Convergent developments: cognitive-developmental correlates of growth in infant/ toddler peer skills. Child Development, 57 (2), 275-286.

Bussab, V. S. R., \& Maluf, M.P.C. (1998). A creche como contexto sócio-afetivo de desenvolvimento: os padrões interacionais e o ajustamento das crianças. Revista Brasileira de Crescimento e Desenvolvimento Humano, 8 (1/2), 33-39.

Campos, M. M., Fullgraf, J., \& Wiggers, V. (2006). A qualidade da educação infantil brasileira: alguns resultados de pesquisa. Cadernos de Pesquisa, 36 (127), 87-128.

Carvalho, A. M., Veiga, C. R., Neves, C. V., Ferreira, D. M. L., Gouveia, D. L., Arruda, F. F., et al. (2006). Projeto caixa de brinquedos: uma análise da capacitação de profissionais de instituições de educação infantil em Minas Gerais, Brasil. Psicologia em Revista, 12 (19), 34-43.

Cole, M., \& Cole, S. R. (2004). O desenvolvimento da criança e do adolescente (4a ed.). Porto Alegre: Artmed. 
Eckerman, C. O., \& Didow, S. M. (1996). Nonverbal imitation and toddler's mastery of verbal means of achieving coordinated action. Developmental Psychology, 32 (1), 141-152.

Fenson, L., \& Ramsay, D. S. (1981). Effects of modeling action sequences on the play of twelve-, fifteen-, and nineteenmonth-old children. Child Development, 52 (6), 1028-1036.

Finkelstein, N. W., Dent, C., Gallacher, K., \& Ramey, C.T. (1978). Social behavior of infants and toddlers in a day-care environment. Developmental Psychology, 14 (3), 257-262.

Hanna, E., \& Meltzoff, A. N. (1993). Peer imitation by toddlers in laboratory, home, and day-care contexts: implications for social learning and memory. Developmental Psychology, 29 (4), 701-710.

Hynes, K., \& Habasevich-Brooks, T. (2008). The ups and downs of child care: Variations in child care quality and exposure across the early years. Early Childhood Research Quarterly, 23 (3), 559-574.

Lampreia, C. (2007). A perspectiva desenvolvimentista para a intervenção precoce no autismo. Estudos de Psicologia. (Campinas), 24 (1), 105-114.

Lima, M. C. M. P., Barbarini, G. C., Gagliardo, H. G. R. G., Arnais, M. A. O., \& Gonçalves, V. M. A. (2004). Observação do desenvolvimento de linguagem e funções auditiva e visual em lactentes. Revista de Saúde Pública, 38 (1), 106-112.

Lordelo, E. R. (1998). Educadores de creche: concepções e práticas. Interação, 2 (1), 113-132.

Lordelo, E. R. (2002). "Agora vá com a tia que a mamãe vem mais tarde": creche como contexto brasileiro de desenvolvimento. In E. R. Lordelo, A. M. A. Carvalho \& S. H. Koller (Orgs.), Infância brasileira e contextos de desenvolvimento (pp.75-95). São Paulo: Casa do Psicólogo.

Lordelo, E. R., \& Carvalho, A. M. A. (2006). Padrões de parceria social e brincadeira em ambiente de creches. Psicologia em Estudo, 11 (1), 99-108.

Lordelo, E. R., Chalhub, A. A., Guirra, R. C., \& Carvalho, C. S. (2007). Contexto e desenvolvimento cognitivo: frequência à creche e evolução do desenvolvimento mental. Psicologia: Reflexão e Crítica, 20 (2), 324-334.

Meneghini, R., \& Campos-de-Carvalho, M. I. (2003). Arranjo espacial na creche: espaços para interagir, brincar isoladamente, dirigir-se socialmente e observar o outro. Psicologia: Reflexão e Crítica, 16 (2), 367-378.

Mueller, E., \& Brenner, J. (1977). The origins of social skills and interaction among playgroup toddlers. Child Development, 48 (5), 854-861.
NICHD Early Child Care Research Network. (2004). Type of child care and children's development at 54 months. Early Childhood Research Quarterly, 19 (1), 203-230.

Palangana, I. C. (2001). Desenvolvimento e aprendizagem em Piaget e Vygotsky: a relevância do social (3a ed.) São Paulo: Summus.

Parten, M. (1932). Social participation among preschool children. Journal of Abnormal and Social Psychology, 27(3), 243-269.

Penn, H., Burton, V., Lloyd, E., Potter, S., Sayeed, Z., \& Mugford, M. (2006). What is known about the long-term economic impact of centre-based early childhood interventions? Technical Report. In Research Evidence in Education Library. London: EPPI-Centre.

Queiroz, N. L. N., Maciel, D. A., \& Branco, A. U. (2006). Brincadeira e desenvolvimento infantil: um olhar sociocultural construtivista. Paidéia, 16 (34), 169-179.

Ramos, C. S.. Pedromônico, M. R. M., Shinzato, A. R., \& Lucas, S. (2002). Comparação do desenvolvimento do comportamento de crianças de creche públicas e particulares no segundo ano de vida. Pró-Fono Revista de Atualização Científica, 14 (3), 401-408.

Rezende, M. A., Lima, F. G., Beteli, V. C., \& Santos, J. L. F. (2003). Habilidades de linguagem e pessoal-social de crianças de 0 a 3 anos de idade cuidadas em creches. Revista Brasileira de Crescimento e Desenvolvimento Humano, 13 (1), 40-52.

Seabra, K. C. \& Seidl de Moura, M. L. (2005). Alimentação no ambiente de creche como contexto de interação nos primeiros dois anos de um bebê. Psicologia em Estudo, 10 (1), 77-86.

Shore, R. (2000). Repensando o cérebro. Porto Alegre: Mercado Aberto.

Souza, T. N. \& Campos-de-Carvalho, M. (2005). Qualidade de ambientes de creches: uma escala de avaliação. Psicologia em Estudo, 10 (1), 87-96.

Vasconcelos, C. R. F., \& Ferreira, M. C. R. (2002). Crianças pequenas brincado em creche: a possibilidade de múltiplos pontos de vista. Estudos de Psicologia, 7 (2), 259-270.

Vigotsky, L. S. (2000). A formação social da mente (6a ed.) São Paulo: Martins Fontes.

Vitta, F. C. F., \& Emmel, M. L. G. (2004). A dualidade cuidado x educação no cotidiano no berçário. Paidéia: Cadernos de Psicologia e Educação, 14 (28), 177-189.

Recebido em: 31/8/2009

Versão final reapresentada em: 11/2/2010

Aprovado em: 18/3/2010 